\title{
Using Off-axis Type Holography for CCD Camera Characterization
}

\author{
E. Voelkl
}

FEI Company, 5350 NE Dawson Creek Drive, Hillsboro, OR 97124-5793

Phase noise and amplitude noise has conventionally been defined as a simple expression given in, e.g., [1-3]. On the other hand, it seems intuitive that once a hologram is digitized, relevant noise levels are well defined. However, as found by simulating off-axis type holograms on a single electron base, the effects of both data acquisition and reconstruction process must be included and it it turns out that the acquisition parameters and reconstruction parameters are key players for the definition of the phase and amplitude noise from a given hologram.

Fig. 1 indicates the generic setup with the intensity distribution at the CCD camera followed by the CCD camera, the digital hologram, the reconstruction process and the final phase/amplitude images with their corresponding noise levels.

There are 2 details to consider closely. First, it is understood that the pixel values found in the digital hologram are not corresponding to a summation of single electron events, i.e., a pixel count of 100 does not represent 100 electrons for that pixel. This is generally true because CCD cameras act as black box and provide, in a simple model, only pixel values that are proportional to the number of electrons. Within the linear range of CCD cameras, this is a fairly good approach. Second, it is assumed that the noise in the intensity distribution to be recorded as well as the noise in the digital hologram can be described as a pure Poisson noise.

With those 2 details in mind, a digital hologram can be simulated for an ideal camera where the modulation transfer function (MTF) and the detection efficiency (DE) are both "1", which of course indicates an unlikely camera that makes the Quantum Mechanical intensity distribution collapse in selected points only, where each point correlates to a pixel of the CCD camera. Anyway, for the ideal camera, a pixel value can be interpreted directly as the number of electrons in that pixel.

The main difference between a simulated hologram and a real (digital) hologram is that the mean number of electrons per pixel is an unknown in the real hologram. However, by reconstructing, e.g., the phase image from both holograms, it becomes fairly easy to find a value that when multiplied with the real hologram converts the pixel values of the real hologram to numbers that closely approximate electron counts. (Specifically for electron holograms recorded with the conventional single biprism setup, Fresnel fringes provide some minor complications which can be minimized by recording two subsequent holograms and using them as hologram and reference-hologram pair.)

Under these circumstances, the effect of the reconstruction process on the noise in the phase and amplitude images can be derived as discussed in [4] and it has been shown that the simulated and experimental results remain in excellent agreement when changing the reconstruction parameters.

So far, the MTF, of course, is missing. However, since the contrast of the interference fringes (all considerations are without an object at this point) is measured from the real hologram, it makes sense to simulate the holograms according to the measured fringe contrast. This is justifiable by the 
fact that the effect of the MTF of CCD cameras is generally described as a constant factor for a given sampling rate $(s)$ and usually depends little on the number of electrons recorded.

By adjusting the mean pixel value of the real hologram via a multiplication factor, the phase noise in both the simulated and real phase images can be matched. Once a match is reached, the pixel values in the real hologram can be interpreted as representing actual electron counts. Furthermore, if it is possible (by e.g., calibration of the current from the fluorescent screen and some geometrical considerations) to determine the number of incident electrons onto the CCD camera during the exposure time, the DE of the real camera can be determined for the given sampling rate. Similarly, if the fringe contrast of the intensity distribution can be approximated by, e.g., using the additional magnification provided by a GIF (with the energy slit out) and measuring the fringe contrast on the GIF-CCD camera, both the MTF and DE can be determined for that camera as a function of $s$.

Then, according to [4], the noise in the phase image is proportional to:

$$
\sim 1 /(\mu \cdot \operatorname{sqrt}[N])
$$

where $N$ corresponds to the mean number of electrons per pixel in the hologram (this is different from earlier definitions) and $\mu(<1)$ corresponds to the fringe contrast in the hologram and $\mu^{*} / \mathrm{MTF}$ $(\mathrm{MTF}<1)$ corresponds to the fringe contrast before the CCD camera. In this case, the effect of the MTF now appears as:

$$
\sim 1 /\left(\mu^{*} \cdot \operatorname{sqrt}\left[N / \mathrm{MTF}^{2}\right]\right)
$$

allowing to characterize a CCD camera in terms of how many electrons are effectively destroyed as a function of the sampling rate or, generally, as a function of the spatial frequencies of a signal. As discussed in [4], at sampling rates $(s)$ of 5 and binning factors of 1 for a $2 \mathrm{k}$ by $2 \mathrm{k}$ camera, more than 95 out of 100 electrons are literally destroyed. This indicates significant potential improvements for CCD cameras especially for off-axis type holography or when used for beam sensitive samples as e.g., in the area of biology.

\section{References}

[1] H.Lichte, et al., Optik 77 (3) (1987).

[2] A. Harscher, H. Lichte, Ultramicroscopy 64 (1996) 57-66.

[3] W.J. deRuijter, J.K. Weiss, Ultramicroscopy 50 (1993).

[4] E. Voelkl, Ultramicroscopy, in print, doi:10.1016/j.ultramic.2009.11.010

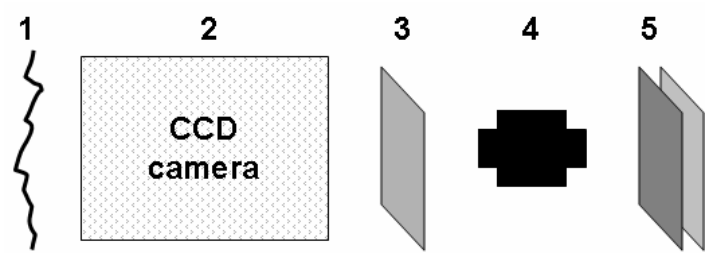

FIG. 1. 1: intensity distribution at CCD; 2: CCD camera; 3: digital hologram with sampling rate $s$; 4: reconstruction process; 5: reconstructed phase and amplitude image. 